\title{
Treino de comportamentos de intimidade para terapeutas em processo de formação em Psicoterapia Analítica Funcional
}

\section{Training intimacy behaviors for therapists in the process of training Functional Analytic Psychotherapy
El entrenamiento de la conducta de intimidad para terapeutas en proceso de formación en Psicoterapia Analítica Funcional

Mariana Sampaio de Almeida', Angela de Loyola e Silva Runnacles ${ }^{2}$, Jocelaine Martins da Silveira ${ }^{3}$ [1] [3] Universidade Federal do Paraná [2] Faculdades de Administração, Ciências, Educação e Letras | Título abreviado: Treino de comportamentos de intimidade
| Endereço para correspondência: I Email: marianasampaiodealmeida93@gmail.com I DOI: 10.18761/pac.2016.020

Resumo: O presente estudo verifica os efeitos de um treino de comportamentos de intimidade no repertório comportamental de terapeutas em formação. O método consiste na aplicação de um treino, com duração de cinco horas, ministrado por uma terapeuta com experiência no ensino da FAP. Os participantes são seis graduandos de Psicologia de uma Universidade. O treino é programado e avaliado quanto ao desenvolvimento de comportamentos ligados ao "saber que" e ao "saber como". A análise dos dados consiste na avaliação de medidas pré-treino e pós-treino, nas quais os participantes descrevem comportamentos de intimidade que um terapeuta FAP pode emitir no contexto terapêutico e participam de um cenário de role-playing de um contexto terapêutico, no qual recebem a tarefa de emitir comportamentos de intimidade. Os resultados indicam que o treino promove melhoras na emissão de comportamentos ligados ao "saber que". Alguma melhora nos comportamentos ligados ao "saber como" é obtida, especialmente no comportamento de dirigir ações sob controle das variáveis relevantes do aqui/agora da sessão, ainda que não de maneira satisfatória. A replicação do treino é encorajada com a utilização de diferentes métodos de avaliação. Os métodos de avaliação de resultados de treinos de terapeutas FAP são discutidos e são fornecidas algumas diretrizes para estudos futuros.

Palavras-chave: treino de terapeutas, intimidade, psicoterapia analítica funcional, supervisão. 
Abstract: This study verifies the effects of a training for intimate behavior in the behavioral repertoire of therapists in training. The method consists of applying a training that lasts five hours, taught by a therapist with experience in the teaching FAP. The participants consist of six undergraduate students of Psychology from an university. The training is planned and evaluated for the development of behaviors linked to "know that" and "know how". Data analysis consists of the evaluation of pre-training measures and post-training measures, in which participants describe behaviors of intimacy that a FAP therapist can deliver in the therapeutic context; and participate in a role-playing scenario of a therapeutic context in which the person is given the task of issuing a behavior of intimacy. The results indicate that the training improve their emission of behaviors linked to "know that". Some improvement in behaviors linked to "know how" has been achieved, especially in the behavior of direct actions under control of the "here now", but not satisfactorily. Replication of the training is encouraged with the use of other evaluation methods. The methods for evaluating the results of FAP therapist training are discussed and some guidelines are provided for future studies.

Keywords: therapist training, intimacy, functional analytic psychotherapy, supervision.

Resumen: El presente estudio verifica los efectos de un entrenamiento de comportamientos de intimidad en el repertorio conductual de terapeutas en formación. El método consiste en la aplicación de un entrenamiento, de cinco horas de duración, impartido por una terapeuta con experiencia en la enseñanza de FAP. Los participantes son seis graduandos de Psicología de una Universidad. El entrenamiento es programado y evaluado con respecto al desarollo de conductas ligadas al "saber qué" y al "saber cómo". El análisis de datos consiste en la evaluación de medidas previas y posteriores al entrenamiento, en las cuales los participantes describen comportamientos de intimidad que un terapeuta FAP puede emitir en un contexto terapeutico y participan de un escenario de role-playing de un contexto terapeutico, en el cual reciben la tarea de emitir comportamientos de intimidad. Los resultados indican que el entrenamiento promueve mejorías en la emisión de comportamientos ligados al "saber qué". Alguna mejoría en los comportamientos ligados al "saber cómo" es obtenida, especialmente en el de dirigir acciones bajo control de las variables importantes de aqui y ahora de la sesión, aunque no sea de una manera satisfactoria. La replicación del entrenamiento es alentada con la utilización de diferentes métodos de evaluación. Esos métodos de evaluación de resultados de entrenamientos de terapeutas FAP son discutidos y fornecidas algunas diretrices para estudios futuros.

Palabras-clave: entrenamiento de terapeutas, intimidad, psicoterapia analítica funcional, supervisión. 
O comportamento de interagir intimamente com outras pessoas frequentemente relaciona-se com a severidade de problemas psicológicos. Abbey, Clopton e Humphreys (2007), por exemplo, conduziram uma pesquisa com pessoas diagnosticadas com transtorno obsessivo-compulsivo (TOC) e que apresentavam grau moderado de estresse em relação ao transtorno $(\mathrm{N}=64)$. Instrumentos que mediam a severidade do transtorno (ObsessiveCompulsive Inventory-Revised, Foa et al., 2002) e a intimidade (Personal Assessment of Intimacy in Relationships, Schaefer \& Olson, 1981) foram preenchidos pelos participantes e os resultados indicaram uma correlação negativa entre as duas variáveis investigadas. Assim, parece que quanto menor o número e/ou intensidade de interações íntimas vivenciadas por uma pessoa com TOC, maior pode ser a severidade do transtorno.

A falta de relacionamentos íntimos também está associada ao desenvolvimento da depressão. Este dado foi encontrado a partir de uma revisão de literatura que examinou se alguns fatores específicos - perda da mãe antes dos 11 anos, mais de três crianças com menos de 14 anos dentro de casa, falta de relacionamentos íntimos e falta de um trabalho fora de casa -, podem indicar risco para o desenvolvimento da depressão (Patten, 1991). Os resultados indicaram que apenas a falta de relacionamentos íntimos foi identificada significativamente como fator de risco para a doença. Há, ainda, outros estudos que indicam a relação entre a falta de intimidade e o desenvolvimento de transtornos psiquiátricos (Newth \& Rackman, 2001; Stemberger, Thomas, Mansueto, \& Carter, 2000; Wetterneck \& Hart, 2012).

O estudo da intimidade, portanto, parece ser indispensável frente a questões de tratamento e prevenção de transtornos psiquiátricos. Uma agenda para o estudo da intimidade prevê a definição precisa sobre os comportamentos nela envolvidos, o desenvolvimento de medidas que a avaliem e de estratégias para seu incremento (Wetterneck \& Hart, 2012).

No que diz respeito a estudos analítico-comportamentais, a definição de intimidade proposta por Cordova e Scott (2001) tem recebido destaque por diversos estudos (Guenzen \& Silveira, 2013; Knott, Wetterneck, Derr, \& Tolentino, 2015; Kohlenberg, Kohlenberg, \& Tsai, 2011; Silva-Dias, 2015; Silva-
Dias \& Silveira, 2016; Sousa \& Vandenberghe, 2007; Wetterneck \& Hart, 2012). Segundo Cordova e Scott (2001), a intimidade se refere a um processo interpessoal, que se desenvolve a partir de uma sequência de eventos nos quais um comportamento interpessoal vulnerável à punição (CIVP) é emitido e, ao invés de ser punido, é reforçado pelo ouvinte/ outro membro da díade.

O CIVP é idiossincrático, uma vez que é definido como o comportamento historicamente punido na vida de uma pessoa (Cordova \& Scott, 2001). Sua emissão envolve o risco característico de qualquer relação de intimidade e, quando reforçada e sucedida por reforços positivos em vez da apresentação da estimulação aversiva, consolida-se uma interação íntima (Kohlenberg, Kohlenberg, \& Tsai, 2011; Vandenberghe \& Pereira, 2005). A reciprocidade nessa interação acontece quando ambos os membros emitem e reforçam CIVPs e há dados que indicam que relações assim estruturadas promovem sentimentos mais intensos de afeição e proximidade entre as pessoas, quando comparadas a relações nas quais apenas uma pessoa emite CIVPs e é reforçada por essa emissão (Sprecher, Treger, Wondra, Hilaire, \& Wallpe, 2013).

Quando a emissão do CIVP é reforçada em uma interação social, os relatos de intimidade e conexão entre as pessoas são mais prováveis. Essa relação foi observada no estudo de Haworth et al. (2015), no qual estudantes de uma Universidade receberam a tarefa de se tornar próximos de um assistente de pesquisa, com o qual passariam algum tempo juntos. Os participantes foram distribuídos aleatoriamente em três grupos: grupo controle $(\mathrm{N}=34)$, grupo da condição de evocar $(\mathrm{N}=32)$ e grupo da condição de evocar e responder $(\mathrm{N}=33)$. Enquanto no grupo controle o tempo passado com o assistente de pesquisa consistia na atividade de assistirem a um vídeo juntos, nos outros grupos os participantes deviam responder a um conjunto de 20 questões pessoais que evocavam CIVPs e que foram realizadas pelos assistentes de pesquisa. $\mathrm{Na}$ condição de evocar, os assistentes de pesquisa foram instruídos a apenas ouvir as respostas dos participantes, sendo objetivos nessa escuta. Já na condição de evocar e responder, eles foram orientados e treinados a fornecer uma resposta natural aos CIVPs dos participantes, com o objetivo de reforçar a sua emissão. 
Antes e depois da intervenção, os participantes preencheram instrumentos que mediram a conexão e proximidade com o pesquisador (Connectedness Scale-Research Assistant, Haworth et al., 2015). Houve uma diferença estatisticamente significativa entre o grupo da condição de evocar e responder e os grupos das demais condições. Os participantes que tiveram como resposta a seus CIVPs o reforço natural se sentiram mais conectados e próximos dos assistentes de pesquisa e os mesmos resultados se mantiveram nas coletas de follow-up $48 \mathrm{~h}$ e 2 semanas após a intervenção. Haworth et al. (2015) sugeriram, portanto, que a resposta natural e reforçadora para a emissão de um CIVP é uma variável significativa que determina a sensação de conexão entre indivíduos.

Há dados que indicam que a própria emissão do CIVP já é importante em uma relação íntima. No estudo de Tsai, Plummer, Kanter, Newring, e Kohlenberg (2010), a emissão de CIVP por uma terapeuta - autorrevelação de informações pessoais - apresentou efeitos positivos para $91 \%$ dos clientes atendidos, estando inclusos em tais efeitos a sensação de proximidade da terapeuta e a confiança no relacionamento terapêutico. Os autores sugeriram que a autorrevelação do terapeuta pode aumentar a intimidade no relacionamento terapêutico. A autorrevelação de eventos privados do terapeuta, por sua vez, ainda não foi avaliada em relação a seus efeitos em comportamentos de intimidade do cliente, mas há dados indicando que a sua emissão evoca comportamentos clínicos de melhora dos clientes em sessão (Guenzen, 2014). Assim, o contexto terapêutico parece ser adequado para a emissão de CIVPs pelo terapeuta (Guenzen, 2014; Tsai et al., 2010). Além disso, a modelagem dos comportamentos de intimidade do cliente pode ser pertinente nesse contexto, dada sua natureza interpessoal e a possibilidade de o terapeuta diretamente observar e intervir sobre os comportamentos do cliente em tempo real (Wetterneck \& Hart, 2012).

Considerando o contexto clínico, a Psicoterapia Analítica Funcional (FAP; Kohlenberg \& Tsai, 1991; Tsai, Kohlenberg, Kanter, Kohlenberg, \& Follette, 2011) é uma terapia privilegiada por focar nos problemas interpessoais do cliente e em questões de intimidade, como a habilidade de confiar profundamente nos outros, ser autêntico e se vulnerabili- zar numa relação (Tsai, Callaghan, \& Kohlenberg, 2013; Wetterneck \& Hart, 2012).

O foco do trabalho de um terapeuta FAP está na evocação e consequenciação de comportamentos clinicamente relevantes (CRBs), comportamentos interpessoais que o cliente expõe no contexto terapêutico, e que podem representar problemas (CRBs1) ou melhoras (CRBs2), de acordo com os objetivos do cliente na terapia. Ainda que os CRBs de cada cliente sejam idiossincráticos, é provável que um cliente com problemas de intimidade apresente CRBs1 com a função de evitar a vulnerabilidade, não revelando informações pessoais ou compartilhando emoções, e CRBs2 com a função de se vulnerabilizar, compartilhando sentimentos, pensamentos e medos (Wetterneck \& Hart, 2012). A resposta natural e contingente do terapeuta aos CRBs é o que possibilita a mudança clínica e há alguns estudos recentes que têm demonstrado isso de maneira sistematizada (Freitas, 2011; Mangabeira, 2014; Meurer, 2011; Oshiro, Kanter \& Meyer, 2012; Villas-Bôas, 2015).

Alguns autores apresentaram contribuições importantes acerca da sistematização dos comportamentos de intimidade que um terapeuta FAP pode emitir no contexto terapêutico. Dentre tais comportamentos, o terapeuta pode emitir CIVPs, reforçar naturalmente a emissão de CIVPs pelo cliente e modelar o comportamento do cliente para que ele reforce os CIVPs do terapeuta (Fugita, 2014; Kohlenberg, Kohlenberg, \& Tsai, 2011). Comportamentos de intimidade do terapeuta também podem ser pensados em termos das cinco regras da FAP: observar CIVPs do cliente (Regra 1), evocar CIVPs (Regra 2), reforçar a sua emissão (Regra 3), verificar o efeito da resposta no comportamento do cliente (Regra 4) e fornecer interpretações funcionais do comportamento de intimidade do cliente (Regra 5) (Wetterneck \& Hart, 2012). Ficar sob controle das variáveis relevantes identificadas na formulação de caso do cliente e das variáveis que controlam o comportamento do cliente e do terapeuta no aqui/agora do contexto terapêutico também são importantes comportamentos de intimidade a serem emitidos pelo terapeuta (Fugita, 2014).

Dentre esses procedimentos, apenas a evocação e o reforço de CIVPs foram estudados quanto a seus efeitos no comportamento de intimidade do cliente 
(Silva-Dias, 2015; Siva-Dias \& Silveira, 2016). No tratamento de um casal com problemas conjugais, duas intervenções foram programadas e apresentadas alternadamente na ordem $\mathrm{ABAB}$, cada qual com duas sessões. Na condição A, a terapeuta utilizou como procedimentos o treinamento de comunicação e a resolução de problemas, enquanto na condição B foi aplicada a FAP com ênfase na evocação e consequenciação dos CIVPs para cada membro do casal. O aumento na emissão de comportamentos de melhora de ambos os parceiros foi mais sensível nas condições B e a emissão de CIVPs de ambos ocorreu somente nas condições B, evidenciando que a ênfase na evocação e consequenciação dos CIVPs pelo terapeuta promove melhoras no repertório de intimidade do cliente.

Ainda que esses resultados sejam promissores, é pertinente o questionamento sobre como terapeutas analítico-comportamentais entendem a intimidade no contexto terapêutico. Guenzen e Silveira (2013) aplicaram um instrumento para terapeutas analítico-comportamentais ( $\mathrm{N}=74)$, a fim de investigar a acepção do termo intimidade por parte dos terapeutas. A maioria dos participantes (89\%) indicou a intimidade como um processo bidirecional, mas respondeu a outras questões do questionário a caracterizando como um processo unidirecional, ou seja, não envolvendo a figura ativa do terapeuta. Mais da metade dos terapeutas (58\%), por exemplo, não apresentou respostas precisas e adequadas quando solicitados a listar três ações terapêuticas que caracterizassem intimidade, seja deixando a questão do questionário sem responder, seja indicando ações do cliente ou ações ligadas a fatores terapêuticos comuns - que se configuram como condições importantes para que a intimidade se estabeleça, mas não como comportamentos próprios que a caracterizam (Guenzen \& Silveira, 2013). Além disso, os participantes que apresentaram respostas precisas (38\%) citaram grande variedade de ações, o que fez com que as autoras sugerissem que há inconsistência na compreensão do fenômeno.

Tendo isso em vista, é pertinente o desenvolvimento de um treino de terapeutas para o comportamento de intimidade no contexto da FAP. Há dados que apoiam a necessidade de desenvolver um treino com esse objetivo, uma vez que terapeutas treinados em FAP apresentam mais comportamentos de inti- midade no contexto terapêutico que terapeutas não treinados em FAP, principalmente no que diz respeito à expressão de sentimentos positivos e à emissão de comportamentos de honestidade e genuinidade (Knott, Wetterneck, Derr, \& Tolentino, 2015). Isso pode ser explicado pela estrutura dos treinos da FAP que, ao enfatizar a tomada de riscos interpessoais do terapeuta e o uso de comportamentos promotores de intimidade, está diretamente associada ao comportamento de intimidade do terapeuta no contexto terapêutico (Knott et al., 2015).

Assim, os comportamentos de intimidade são passíveis de serem treinados pela Psicoterapia Analítica Funcional, uma vez que para que ela seja bem conduzida torna-se importante o conhecimento dos princípios dessa terapia e a emissão de comportamentos que envolvam a tomada de risco corajosa e estratégica do terapeuta (Kanter et al., 2012). Os supervisionandos em FAP são, portanto, estimulados a desenvolver comportamentos ligados a um "saber que" e a um "saber como", que se caracterizam, respectivamente, por apresentar repertório verbal sobre os acontecimentos da terapia e efetivamente perceber, evocar e fortalecer os CRBs dos clientes no contexto terapêutico (Tsai, Callaghan, Kohlenberg, Follette, \& Darrow, 2011).

Os estudos sobre treino de terapeutas e supervisão na FAP ainda estão em fase inicial. Há estudos que utilizaram a relação entre supervisor e supervisionando para ilustrar momentos de aprendizagem proporcionados pela supervisão (Sousa \& Vandenberghe, 2007; Wielenska \& Oshiro, 2012) ou para verificar seus efeitos nos comportamentos do supervisionando e do cliente em sessão (Lepienski, 2015). Alguns utilizaram técnicas de feedback sobre o desempenho do terapeuta em sessão (Follette \& Callaghan, 1995; Meurer, 2011) e há ainda um estudo que avaliou os efeitos de uma supervisão FAP na identificação de CRBs por um terapeuta (Silveira et al., 2009). Nesse estudo, foi realizada a modelagem direta pelo supervisor, no aqui-agora da supervisão, de comportamento interpessoais relevantes do supervisionando, associada ao preenchimento do instrumento FIAT-T (Functional Idiographic Assessment Template - Therapist, Callaghan, 2006), que investiga padrões de comportamento interpessoal do terapeuta. Por fim, Kanter et al. (2012) avaliaram os efeitos de um treino FAP, composto por exercícios 
experienciais, apresentações didáticas e discussões de caso, no comportamento de reforçar naturalmente os comportamentos do cliente em sessão.

Apenas dois estudos relataram treino direcionado a vários terapeutas concomitantemente (Kanter et al., 2012; Wielenska \& Oshiro, 2012), enquanto que outros estudos consistiram em supervisões realizadas entre um supervisor e um supervisionando (Follette \& Callaghan, 1995; Lepienski, 2015; Meurer, 2011; Silveira et al., 2009; Sousa \& Vandenberghe, 2007). A supervisão ou treino destinado a apenas um supervisionando não é um problema. Contudo, a possibilidade de treinar mais terapeutas no mesmo período de tempo em que se treina apenas um parece importante, e é notável que essa alternativa tenha sido pouco desenvolvida.

A metodologia com a qual os resultados de um treino ou supervisão são mensurados também merece destaque na discussão de estudos sobre treino e supervisão de terapeutas. Há, por exemplo, um estudo que não coletou dados sobre a efetividade da intervenção (Follette \& Callaghan, 1995) e outro que relatou seus resultados de maneira descritiva, com base em observações não sistemáticas (Wielenska \& Oshiro, 2012). Análises qualitativas de trechos de uma supervisão gravada também foram realizadas (Sousa \& Vandenberghe, 2007), essas, contudo, pouco revelaram sobre mudanças observadas no repertório dos participantes com a supervisão, uma vez que se propuseram a refletir sobre a aplicação dos princípios da FAP no contexto da supervisão.

Kanter et al. (2012), por sua vez, avaliaram os resultados de um treino para terapeutas interessados no manejo da FAP $(\mathrm{N}=16)$ por meio da FAPIS (FAP Impact Scale, Kanter et al., 2012) - escala que avalia o impacto de um treino FAP -, e por meio de vinhetas e questionários pessoais. As vinhetas contiveram interações hipotéticas entre clientes e terapeutas, perante as quais o terapeuta deveria escrever sobre como agiria. As medidas foram aplicadas antes e após o treino. Os autores ressaltaram importantes limitações dessas medidas, uma vez que se basearam em autorrelatos (FAPIS e questionários pessoais) e em situações hipotéticas (vinhetas), que podiam não corresponder ao que o terapeuta efetivamente faz no contexto terapêutico com seu cliente. Assim, os autores recomendaram que os próximos estudos priorizassem a medida do comportamento dos participantes no contexto terapêutico, por meio da gravação e análise das sessões conduzidas pelos supervisionandos. A mesma recomendação foi realizada por Knott et al. (2015).

Essa alternativa de avaliação dos resultados foi utilizada por Meurer (2011) e Lepienski (2015). Os autores utilizaram a FAPRS (Functional Analytic Psychotherapy Rating Scale, Callaghan \& Follette, 2008), uma escala de categorização de sessões da FAP, para categorizar e analisar sessões conduzidas por um supervisionando, a fim de verificar mudanças no repertório dele após as sessões de supervisão. É necessário ressaltar, contudo, que Meurer (2011) aplicou e replicou o procedimento para duas díades terapeuta-cliente, enquanto Lepienski (2015) o aplicou para uma tríade supervisor-supervionando-cliente. Tendo isso em vista, cabe o questionamento sobre a viabilidade de medir o comportamento do terapeuta no contexto terapêutico quando se trata de um treino com vários participantes, visto que as sessões de cada participante deveriam ser gravadas, transcritas, categorizadas e analisadas. Kanter et al. (2012) já fizeram esse questionamento, e apontaram como possível alternativa o uso de cenários de role-playing, que podem ser importantes medidas substitutivas dos resultados da terapia (Fairburn \& Cooper, 2011), ainda que menos desejáveis que as medidas precisas do comportamento do terapeuta em sessão.

As regras para aplicação da FAP exigem que o terapeuta emita comportamentos de intimidade no contexto terapêutico, tornando especial o treino de terapeutas para a competência no uso desta estratégia. Tendo isso em vista, o presente estudo teve o objetivo de verificar os efeitos de um treino de comportamentos de intimidade no repertório comportamental de terapeutas em formação. A primeira hipótese do estudo admite que o treino influencia os comportamentos de "saber que" dos participantes, isto é, a capacidade de sistematizar comportamentos de intimidade que o terapeuta FAP pode emitir no contexto terapêutico. A segunda hipótese admite que o treino influencia a emissão de comportamentos de intimidade pelo terapeuta FAP no contexto terapêutico, quais sejam: dirigir ações sob controle das variáveis relevantes do aqui/agora da sessão, tatear eventos privados no contexto terapêutico e reforçar naturalmente a emissão de CIVPs pelo cliente. 


\section{Método}

\section{Participantes}

Os participantes foram seis alunos do quarto ano do curso de Psicologia de uma Universidade pública brasileira, os quais estavam iniciando seus primeiros atendimentos clínicos na perspectiva da Análise do Comportamento, e não tinham experiências anteriores com a prática clínica. Os critérios de seleção para os participantes foram: a) já ter cursado uma disciplina do currículo do curso de Psicologia de uma Universidade Pública que abordava os princípios da Psicoterapia Analítica Funcional; b) não possuir experiência clínica ou possuir no máximo um mês de experiência clínica supervisionada na perspectiva da Análise do Comportamento.

\section{Colaboradores}

Os colaboradores do estudo foram: uma treinadora qualificada - que aplicou o treino-; uma atriz que dramatizou o papel de cliente nos cenários de role-playing pré-treino e pós-treino; e dois juízes que avaliaram o desempenho dos participantes nos cenários de role-playing. A treinadora foi uma terapeuta com 15 anos de experiência clínica e um ano de experiência como supervisora FAP, e que lecionava aulas de Análise do Comportamento em uma faculdade de Psicologia. Os demais colaboradores foram alunos do quinto ano do curso de Psicologia de uma Universidade pública. Os colaboradores que atuaram como juízes já haviam realizado estudos de decomposição de comportamentos no contexto clínico com uso da FAP, eram habilitados a realizar a categorização na escala da FAP (Functional Analytic Psychotherapy Rating Scale, Callaghan \& Follette, 2008) e tinham um ano de estágio supervisionado em FAP.

\section{Instrumentos e materiais}

Filmadora para gravar os cenários de role-playing pré-treino e pós-treino.

Notebook para exibir os cenários de role-playing pré-treino e pós-treino aos juízes.

\section{Local}

Sala de aula do Departamento de Psicologia de uma Universidade pública, reservada para a execução do treino. Os cenários de role-playing pré-treino e pós-treino e suas avaliações ocorreram na mesma sala, em horários diferentes.

\section{Procedimentos}

Fase I - Medidas coletadas antes do treino: avaliação dos comportamentos ligados ao "saber que" e ao "saber como"

Duas medidas foram coletadas antes do treino, uma para avaliar os comportamentos ligados ao "saber que" e outra para avaliar os comportamentos ligados ao "saber como". Uma descrição detalhada e exemplificada dos comportamentos constituintes das classes "saber que" e "saber como" pode ser visualizada na Tabela 1.

A avaliação dos comportamentos da classe "saber que" foi realizada por meio da aplicação de um instrumento imediatamente antes do início do treino. Os participantes receberam uma folha de registro e foram orientados a preenchê-la. Na folha, constava a seguinte instrução: "Por favor, indique quais são os comportamentos de intimidade que um terapeuta FAP pode emitir no contexto terapêutico para estabelecer a intimidade com o cliente".

A avaliação dos comportamentos da classe "saber como" foi realizada por meio da atuação dos participantes em um cenário de role-playing, que aconteceu no dia anterior ao treino. Foi solicitado que os participantes atuassem em um cenário de role-playing de um contexto terapêutico, no qual desempenhariam o papel de terapeuta FAP. Eles foram orientados a emitir comportamentos de intimidade nesse contexto. Apenas essa instrução foi fornecida, não havendo qualquer orientação sobre quais são os comportamentos de intimidade que um terapeuta pode emitir em sessão. Para isso, receberam uma breve Formulação de Caso (identificação do cliente, da queixa e dos CRBs e uma breve história de vida) de uma cliente com queixas de intimidade. A cliente era atendida na clínica escola da Universidade, pela colaboradora que atuou como cliente nos cenários de role-playing. $\mathrm{O}$ caso foi adaptado a fim de garantir as questões de sigilo que envolviam a cliente. Os participantes tinham, no máximo, dez minutos para desempenhar a tarefa. Os cenários de role-playing foram gravados. 
Tabela 1

Definição exemplificada de comportamentos das classes "saber que" "e saber como"

\begin{tabular}{|c|c|c|}
\hline & "Saber que" & "Saber como" \\
\hline Definição & $\begin{array}{l}\text { Conhecimento intelectual do supervisionando. } \\
\text { Repertório verbal que possibilita descrever } \\
\text { as características importantes do processo } \\
\text { terapêutico. }\end{array}$ & $\begin{array}{l}\text { Conhecimento emocional do supervisio- } \\
\text { nando. É essencialmente o que o terapeu- } \\
\text { ta faz e possui correspondência com o } \\
\text { comportamento modelado pelas contin- } \\
\text { gências. }\end{array}$ \\
\hline $\begin{array}{l}\text { Exemplos: } \\
\text { Reforçar naturalmente CIVPs }\end{array}$ & $\begin{array}{l}\text { Relatar verbalmente a definição de CIVPs, in- } \\
\text { dicando as contingências que os estabelecem } \\
\text { e mantêm; } \\
\text { Relatar verbalmente topografias possíveis para } \\
\text { o reforço natural de CIVPs no contexto clínico; }\end{array}$ & $\begin{array}{l}\text { No aqui/agora da sessão ou supervisão/ } \\
\text { treino, reforçar naturalmente os CIVPs do } \\
\text { cliente ou do supervisor/supervisionando } \\
\text { de maneira adequada e eficaz. }\end{array}$ \\
\hline $\begin{array}{l}\text { Exemplos: } \\
\text { Tatear eventos privados no } \\
\text { contexto terapêutico }\end{array}$ & $\begin{array}{l}\text { Identificar verbalmente as funções do tato } \\
\text { de eventos privados no contexto terapêutico } \\
\text { (reforço natural e/ou evocação de CIVPs); } \\
\text { Identificar verbalmente momentos adequados } \\
\text { e topografias possíveis, de acordo com o } \\
\text { caso; }\end{array}$ & $\begin{array}{l}\text { No aqui/agora da sessão ou supervisão/ } \\
\text { treino, emitir tatos de eventos privados } \\
\text { para o cliente ou para o supervisor/super- } \\
\text { visionando de maneira adequada e eficaz. }\end{array}$ \\
\hline
\end{tabular}

Nota: CIVPs - Comportamentos interpessoais vulneráveis à punição. Definição das classes "saber que" e "saber como" Retirada de Tsai et al. (2011).

Além da tarefa de emitir comportamentos de intimidade, os participantes foram orientados a informar a cliente, em algum momento do role-playing, que não poderiam atendê-la na semana seguinte. Três participantes forneceram como motivo para a cliente a ida a um Congresso de Psicologia, enquanto os outros três informaram período de férias. Essa instrução foi necessária para garantir que a atriz, no papel de cliente, tivesse oportunidades suficientes para emitir comportamentos interpessoais vulneráveis à punição (CIVP) - que, de acordo com a Formulação de Caso fornecida aos participantes, eram qualquer expressão de afeto direcionada a figura do terapeuta. Por exemplo, diante da fala dos terapeutas sobre a interrupção das sessões devido ao período de férias, a atriz no papel de cliente emitia frases como "Mas como vou ficar tanto tempo sem te ver? Eu preciso conversar com você toda a semana, apenas aqui me sinto bem". A escolha dos motivos apresentados pelos terapeutas em treino (Congresso ou período de férias) foi baseada no próprio atendimento que a colaboradora, no papel de atriz, realizava na clínica-escola da Universidade.

A atriz foi instruída a iniciar os cenários de role-playing apresentando relatos sobre alguma dificuldade diária da sua vida, acompanhada da descrição de emoções sobre tal dificuldade. Além disso, ela foi instruída a emitir CIVPs sempre que conseguisse.

\section{Fase II - Aplicação do treino de intimidade}

O treino teve duração de cinco horas e foi programado para permitir que os participantes desenvolvessem os seguintes comportamentos: 1) Ficar sob controle das variáveis relevantes identificadas na formulação de caso do cliente; 2) Modelar comportamentos de intimidade do cliente; 3) Identificar CIVPs; 4) Dirigir ações sob controle das variáveis relevantes do aqui/agora da sessão; 5) Tatear eventos privados nesse contexto; 6) Reforçar naturalmente a emissão de CIVPs pelo cliente. Os procedimentos metodológicos utilizados para o ensino desses comportamentos variaram entre breves apresentações teóricas, exercícios experienciais e teóricos e estudo de caso. Cada exercício experiencial durou cinco minutos e foi realizado em duplas. Esses exercícios deveriam ser realizados duas vezes, a fim de que ambos os membros das duplas pudessem realizar as atividades propostas, invertendo seus papeis. Uma descrição mais detalhada dos procedimentos metodológicos utilizados no treino pode ser visualizada na Tabela 2. 


\section{Tabela 2 \\ Procedimentos Metodológicos Utilizados no Treino}

\begin{tabular}{|c|c|}
\hline Comportamento Objetivo & Estratégia de Ensino \\
\hline $\begin{array}{l}\text { Ficar sob controle das variáveis re- } \\
\text { levantes identificadas na formulação } \\
\text { de caso do cliente }\end{array}$ & $\begin{array}{l}\text { Apresentação de Formulação de Caso da FAP com cliente que possuía queixas de } \\
\text { intimidade. Exercício teórico de previsão de topografias para os CIVPs do cliente e } \\
\text { planejamento de respostas terapêuticas para a sua emissão. }\end{array}$ \\
\hline Identificar CIVPs & Apresentação teórica \\
\hline Modelar o comportamento & $\begin{array}{l}\text { Em duplas, um participante recebeu a tarefa de falar sobre "O que na minha vida contri- } \\
\text { buiu para eu estar aqui?", enquanto o outro deveria modelar o comportamento verbal de } \\
\text { seu colega visando um comportamento objetivo (ex: detalhamento dos fatos; relatos de } \\
\text { eventos frustrantes e etc.). }\end{array}$ \\
\hline $\begin{array}{l}\text { Dirigir ações sob controle das } \\
\text { variáveis relevantes do aqui/agora } \\
\text { da sessão }\end{array}$ & $\begin{array}{l}\text { Em duplas, um participante recebeu a tarefa "Fale sobre o seu dia de hoje", enquanto } \\
\text { o outro deveria trazer o relato do seu colega para o aqui-agora. O instrutor auxiliou os } \\
\text { participantes a usarem frases específicas (ex:"Como você está se sentindo contando } \\
\text { isso aqui para mim?") }\end{array}$ \\
\hline Reforçar naturalmente CIVPs & $\begin{array}{l}\text { As duplas foram instruídas a fazer o exercício de conexão do } 1 \text { minuto. As duplas fica- } \\
\text { ram em silêncio, enquanto se olhavam nos olhos, e procuravam desviar qualquer aten- } \\
\text { ção de outro evento que não a existência de seu par no aqui-agora. No fim, foi orientado } \\
\text { para que expressassem amor e vulnerabilidade com o olhar, além de respeito pelo que o } \\
\text { outro desejava fazer naquele momento do aqui-agora. }\end{array}$ \\
\hline $\begin{array}{l}\text { Tatear eventos privados no contexto } \\
\text { terapêutico }\end{array}$ & $\begin{array}{l}\text { As duplas foram instruídas a fazer uma revelação que dizia respeito à interação com o } \\
\text { parceiro da dupla. O instrutor ajudou fornecendo frases como: "o que sinto agora é..." e } \\
\text { etc. Foi instruído que a revelação deveria ser acolhida pelo outro membro da dupla por } \\
\text { meio de expressões não verbais (olhar, gestos e etc.). }\end{array}$ \\
\hline
\end{tabular}

Nota: FAP - Psicoterapia Analítica Funcional. CIVP - Comportamento interpessoal vulnerável à punição. Formulação de Caso da FAP com cliente com queixas de intimidade - Retirada de Freitas (2011).

Fase III - Medidas coletadas após o treino: avaliação dos comportamentos ligados ao "saber que" e ao "saber como"

Os mesmos procedimentos da Fase I foram adotados: uma avaliação dos comportamentos da classe "saber que", aplicada imediatamente após o treino, e uma avaliação dos comportamentos da classe "saber como", aplicada quatro dias após o treino.

A avaliação dos comportamentos da classe "saber como" também foi realizada por meio de um cenário de role-playing, que seguiu as mesmas diretrizes do cenário de role-playing descrito na Fase I, exceto por um detalhe na tarefa fornecida aos participantes. Assim como no cenário de role-playing pré-treino, os participantes deveriam informar a cliente que não poderiam atendê-la na semana seguinte devido a um Congresso de Psicologia ou devido à entrada no período de férias. Nos cenários de role-playing pós-treino ocorreu uma inversão desses motivos. Os três participantes que informaram sobre o Congresso no cenário de role-playing pré-treino, por exemplo, receberam a tarefa de informar sobre o período de férias no cenário de role-playing pós-treino. Esse procedimento foi adotado a fim de garantir alguma discrepância entre os cenários de role-playing e minimizar efeitos de aprendizagem prévia.

Nessa Fase, também foram adotados procedimentos específicos quanto à avaliação dos cenários de role-playing. Foi sorteado o participante a ser avaliado e a ordem de apresentação das gravações de role-playing que ele participou. Assim, os dois juízes que avaliaram os cenários de role-playing puderam comparar o desempenho de um mesmo participante, sendo cegos em relação ao momento dos cenários de role-playing que ele participou pré-treino ou pós-treino.

Os juízes, hábeis na categorização em FAPRS e treinados para análise e derivação de comportamentos da FAP (ambos realizaram trabalhos de decomposição de comportamentos da FAP), apenas foram orientados a avaliar os seguintes comportamentos do terapeuta em treino: dirigir ações sob controle das variáveis relevantes do aqui/agora 
da sessão do contexto terapêutico; tatear eventos privados nesse contexto; e reforçar naturalmente a emissão de CIVPs pelo cliente. Todos os comportamentos foram avaliados de acordo com a seguinte escala: não emitiu o comportamento (nota 0 ); emitiu o comportamento aquém do necessário (nota 1); emitiu o comportamento na medida do necessário (nota 2). As notas foram atribuídas pelos juízes ao final da visualização de cada cenário de role-playing.

\section{Análise dos dados}

Em relação aos comportamentos ligados ao "saber que", foram comparados os registros de comportamentos de intimidade que um terapeuta FAP pode emitir no contexto terapêutico. A comparação foi realizada entre os registros de pré-treino e pós-treino de todos os participantes. A análise realizada buscou identificar quantos comportamentos foram mencionados e classificá-los como condizentes ou não a comportamentos de intimidade do terapeuta FAP. Os critérios utilizados para determinar se os comportamentos citados diziam respeito a comportamentos de intimidade foram baseados na literatura sobre comportamentos de intimidade do terapeuta FAP no contexto terapêutico (Fugita, 2014; Kohlenberg, Kohlenberg, \& Tsai, 2011; Wetterneck \& Hart, 2012).

Em relação aos comportamentos ligados ao "saber como", foram somadas as notas que cada juiz atribuiu aos três comportamentos avaliados nos cenários de role-playing pré-treino e pós-treino. Cada comportamento recebeu até, no máximo, nota 2 - no caso de os juízes terem considerado que a sua emissão no cenário de role-playing ocorreu na medida do necessário. Assim, a soma das notas de cada juiz variou de zero (nenhum comportamento foi emitido) a seis (todos os comportamentos foram emitidos na medida do necessário) para cada cenário de role-playing. As somas das notas de cada juiz foram dispostas na Tabela 3, separadas por participante e por momento do cenário de role-playing (pré-treino ou pós-treino). Na última coluna da tabela, foi registrado se os dois juízes consideraram que os comportamentos - e quais melhoraram, pioraram ou foram apresentados no mesmo nível nos dois momentos dos cenários de role-playing. Essa avaliação só foi registrada no caso de os dois juízes concordarem em seus pontos de vista, ainda que não no mesmo grau. Por exemplo, os dois juízes concordaram que o comportamento $\mathrm{x}$ não foi emitido por um participante no cenário de role-playing pré-treino, mas foi emitido no cenário de role-playing pós-treino. Os dois juízes concordaram, portanto, que houve uma melhora no desempenho do participante, ainda que um juiz tenha considerado que o comportamento foi emitido aquém do necessário, enquanto o outro considerou que a emissão ocorreu na medida do necessário.

\section{Resultados}

Quanto aos comportamentos da classe "saber que", dos seis participantes, quatro citaram mais comportamentos no registro pós-treino, um citou a mesma quantidade de comportamentos em ambos os registros e um citou menos comportamentos no registro pós-treino. A análise das respostas permitiu a identificação de quatro categorias: a) comportamentos que representam fatores terapêuticos comuns - empatia, aceitação incondicional, consideração positiva e calor humano; b) comportamentos de intimidade do terapeuta FAP; c) comportamentos de intimidade do terapeuta FAP descritos funcionalmente e d) descrição do que a intimidade demanda emocionalmente do terapeuta FAP.

Dos 23 comportamentos citados nos registros pré-treino, 14 representaram fatores terapêuticos comuns - empatia, calor humano, consideração positiva - e nove representaram um dos comportamentos de intimidade do terapeuta FAP - a autorrevelação. Nenhum participante descreveu funcionalmente os comportamentos de intimidade do terapeuta FAP ou descreveu o que a intimidade demanda emocionalmente do terapeuta FAP.

Dos 30 comportamentos citados nos registros pós-treino, cinco representaram fatores terapêuticos comuns e 18 foram classificados como comportamentos de intimidade do terapeuta FAP. Além disso, sete comportamentos do terapeuta FAP foram descritos funcionalmente e sete descrições do que a intimidade demanda emocionalmente do terapeuta FAP foram identificadas.

Exemplos dos comportamentos citados nos registros pré-treino e pós-treino são apresentados na Tabela 3. 


\section{Tabela 3}

\section{Exemplos de Comportamentos Citados nos Registro Pré-Treino e Pós-Treino}

\begin{tabular}{ll}
\hline Características do Registro & Exemplos \\
\hline $\begin{array}{l}\text { Comportamentos que representam } \\
\text { fatores terapêuticos comuns }\end{array}$ & $\begin{array}{l}\text { "Demonstrar empatia em relação à experiência do cliente, seus sentimentos e com- } \\
\text { portamentos" (comportamento registrado no pré-treino). }\end{array}$ \\
$\begin{array}{l}\text { Comportamentos de intimidade do } \\
\text { terapeuta FAP }\end{array}$ & $\begin{array}{l}\text { "O terapeuta deve evocar comportamentos vulneráveis do cliente, modelar o com- } \\
\text { portamento do cliente para reforçar os comportamentos vulneráveis do terapeuta e } \\
\text { reforçar o aparecimento de comportamentos vulneráveis do cliente durante a sessão" } \\
\text { (comportamento registrado no pós-treino). }\end{array}$ \\
$\begin{array}{ll}\text { Comportamentos de intimidade do te- } \\
\text { rapeuta FAP descritos funcionalmente }\end{array}$ & $\begin{array}{l}\text { "Expressar sentimentos em relação aos relatos do cliente e à própria relação terapêu- } \\
\text { tica para permitir ao cliente reforçar esses comportamentos do terapeuta e aumentar } \\
\text { as chances de ele emitir essas respostas fora da sessão" (comportamento registrado } \\
\text { no pós-treino). }\end{array}$ \\
$\begin{array}{ll}\text { Descrição do que a intimidade deman- } \\
\text { da emocionalmente do terapeuta FAP. }\end{array}$ & $\begin{array}{l}\text { "A intimidade demanda disposição por parte do terapeuta de ser íntimo. A intimida- } \\
\text { como aumentar a intimidade. Entretanto, acho que isso seria desnecessário, pois, } \\
\text { para mim, ficou muito claro que o mais importante é querer viver aquela relação e se } \\
\text { deixar ser vulnerável. É necessária uma coragem absurda para isso. Se mostrar para } \\
\text { o outro demanda muito" (comportamento registrado no pós-treino). }\end{array}$ \\
\hline
\end{tabular}

Nota: FAP - Psicoterapia Analítica Funcional.

No que diz respeito às mudanças individuais de cada participante, o número de vezes em que comportamentos de intimidade do terapeuta FAP foram citados aumentou para todos os participantes no registro pós-treino. Os participantes $3,4 \mathrm{e}$ 6 citaram, no registro pós-treino, menos comportamentos representativos de fatores terapêuticos comuns, enquanto os participantes 1,2 e 5 citaram a mesma quantidade desses comportamentos nos dois registros. Os comportamentos de intimidade do terapeuta FAP descritos funcionalmente aumentaram no registro pós-treino dos participantes 2, 3 e 4 e se mantiveram iguais nos registros pré e pós-treino dos participantes 1, 5 e 6 . Os participantes 5 e 6 fizeram descrições após o treino sobre o que a intimidade demanda emocionalmente do terapeuta FAP.

Quanto aos comportamentos ligados ao "saber como", os juízes concordaram nas avaliações de quatro participantes. Os participantes 1,3 e 4 haviam melhorado seus desempenhos no role-playing pós-treino, enquanto o participante 2 manteve seu desempenho constante nos dois momentos do role-playing.

O comportamento de dirigir ações sob controle das variáveis relevantes do aqui/agora da sessão foi aperfeiçoado no role-playing pós-treino pelos três participantes que apresentaram melhoras de acordo com os juízes. O comportamento de tatear eventos privados no contexto terapêutico, por sua vez, foi aperfeiçoado apenas pelo participante 4 , enquanto o comportamento de reforçar naturalmente a emissão de CIVPs pelo cliente foi aperfeiçoado apenas pelo participante 1 .

De acordo com a avaliação dos juízes, o participante 2 emitiu todos os comportamentos desejáveis nos cenários de role-playing pré-treino e pós-treino.

Em relação ao participante 5, os juízes não concordaram com as suas avaliações, uma vez que o juiz 1 considerou que o desempenho do participante foi equivalente nos cenários de role-playing pré-treino e pós-treino, enquanto o juiz 2 considerou que houve uma melhora nos comportamentos de tatear eventos privados no contexto terapêutico e reforçar naturalmente a emissão de CIVPs pelo cliente.

Os juízes também não concordaram em relação ao desempenho do participante 6 , uma vez que o juiz 1 o considerou equivalente nos cenários de role-playing pré-treino e pós-treino, enquanto o juiz 2 considerou que houve piora na emissão do comportamento de reforçar naturalmente a emissão de CIVPs pelo cliente. Os dados de cada participante estão apresentados na Tabela 4. 
Tabela 4

Comportamentos Apresentados nos cenários de role-playing

\begin{tabular}{|c|c|c|c|c|}
\hline Juiz & Participante & $\begin{array}{l}\text { Notas RP } \\
\text { Pré-treino }\end{array}$ & $\begin{array}{l}\text { Notas RP } \\
\text { Pós-treino }\end{array}$ & Avaliação \\
\hline Juiz 1 & Participante 1 & 2 & 4 & $\begin{array}{l}\text { Comportamentos } 1 \text { e } \\
3 \text { melhoraram no RP } \\
\text { pós-treino }\end{array}$ \\
\hline Juiz 2 & Participante 1 & 2 & 6 & \\
\hline Juiz 2 & Participante 2 & 6 & 6 & $\begin{array}{l}\text { A emissão dos compor- } \\
\text { tamentos se manteve } \\
\text { constante }\end{array}$ \\
\hline Juiz 1 & Participante 3 & 0 & 2 & $\begin{array}{l}\text { Comportamento } 1 \text { me- } \\
\text { Ihorou no RP pós-treino }\end{array}$ \\
\hline Juiz 2 & Participante 3 & 1 & 2 & \\
\hline Juiz 1 & Participante 4 & 0 & 2 & $\begin{array}{l}\text { Comportamentos } 1 \text { e } \\
2 \text { melhoraram no RP } \\
\text { pós-treino }\end{array}$ \\
\hline Juiz 1 & Participante 5 & 1 & 1 & $\begin{array}{l}\text { Não houve concordân- } \\
\text { cia na avaliação dos } \\
\text { juízes }\end{array}$ \\
\hline Juiz 1 & Participante 6 & 4 & 4 & $\begin{array}{l}\text { Não houve concordân- } \\
\text { cia na avaliação dos } \\
\text { juízes }\end{array}$ \\
\hline
\end{tabular}

Notas: RP - Role-Playing. Comportamento 1 - Dirigir ações sob controle das variáveis relevantes do aqui/agora da sessão. Comportamento 2 - Tatear eventos privados no contexto terapêutico. Comportamento 3 - Reforçar naturalmente a emissão dos comportamentos interpessoais vulneráveis à punição pelo cliente.

\section{Discussão}

O objetivo do presente estudo foi verificar os efeitos de um treino de comportamentos de intimidade no repertório comportamental de terapeutas em formação. A primeira hipótese admitiu que o treino influenciaria os comportamentos do participante de sistematizar a intimidade que o terapeuta FAP pode emitir no contexto terapêutico. Os resultados indicaram que os participantes registraram após o treino mais comportamentos de intimidade do terapeuta FAP, menos comportamentos que representam fatores terapêuticos comuns e mais descrições funcionais dos comportamentos de intimidade do terapeuta FAP.
Pareceu haver uma similaridade no entendimento acerca da intimidade que os participantes do presente estudo tinham antes da realização do treino e que os terapeutas analítico-comportamentais $(\mathrm{N}=74)$ do estudo de Guenzen e Silveira (2013) apresentaram. Nos dois estudos, os participantes citaram comportamentos que representam fatores terapêuticos comuns como comportamentos de intimidade, deixando pistas de que pode haver pouca compreensão, por parte de uma comunidade mais ampla de terapeutas, das características comportamentais da intimidade. 
A escassa discussão sobre a intimidade na Análise do Comportamento motivou Cordova e Scott (2001) a formularem uma definição comportamental dela. Segundo os autores, há consideráveis limitações no estudo da intimidade pelos analistas do comportamento, uma vez que aqueles que se propuseram a estudá-la apresentaram definições confusas ou limitadas do fenômeno, enquanto o restante evitou o seu estudo por considerá-la um construto hipotético ao invés de um fenômeno comportamental. O presente estudo sugeriu, portanto, que um treino de terapeutas para comportamentos de intimidade pode promover mais clareza sobre os processos comportamentais envolvidos em umainteração íntima.

Outro aspecto a ser observado é que dois dos participantes descreveram, em seus registros pós-treino, o que a intimidade demanda emocionalmente do terapeuta, ainda que as instruções desse registro não solicitassem esse tipo de reflexão. Isto sugere que o treino de intimidade impactou sensivelmente os participantes.

A contagem de comportamentos mencionados anteriormente e posteriormente ao treino expressa apenas parcialmente a aprendizagem relevante para o presente estudo. A participante 6, por exemplo, citou menos comportamentos no registro pós-treino, mas a partir de seu registro é notável alguma aprendizagem emocional sobre a intimidade, uma vez que mencionou “(...) Eu poderia escrever todos os passos aprendidos durante o curso de como aumentar a intimidade. Entretanto, acho que isso seria desnecessário, pois, para mim, ficou muito claro que o mais importante é querer viver aquela relação e se deixar ser vulnerável. É necessária uma coragem absurda para isso. (...)".

Kanter et al. (2012) salientaram que vivências de acolhimento e reforço da tomada de risco e da vulnerabilidade emocional são aspectos importantes no treino de terapeutas. Knott et al. (2015), por sua vez, evidenciaram que algumas características do treino em FAP - o encorajamento de autenticidade no relacionamento terapêutico e o encorajamento para riscos interpessoais - estão associadas com a intimidade do terapeuta no contexto terapêutico. O presente estudo, portanto, conteve vivências indicadas pela literatura como importantes e como associadas ao comportamento de intimi- dade do terapeuta no contexto terapêutico, e seus resultados indicaram que essas vivências podem promover a sensibilização sobre a complexidade emocional envolvida na emissão de comportamentos de intimidade pelo terapeuta.

A segunda hipótese do estudo assumiu que o treino influenciaria melhoras na emissão de comportamentos de intimidade pelos participantes no contexto terapêutico, quais sejam: dirigir ações sob controle das variáveis relevantes do aqui/agora da sessão, tatear eventos privados nesse contexto e reforçar naturalmente a emissão de CIVPs pelo cliente. A hipótese não pôde ser confirmada, uma vez que os juízes que avaliaram o desempenho dos participantes nos cenários de role-playing concordaram que metade dos participantes apresentou melhoras no comportamento de dirigir ações sob controle das variáveis relevantes do aqui/agora da sessão do contexto terapêutico e que apenas um participante apresentou melhoras nos comportamentos de tatear eventos privados e outro no comportamento de reforçar naturalmente a emissão de CIVPs pelo cliente.

Ressalta-se, contudo, que esses resultados podem ser função das características do role-playing. O comportamento de tatear eventos privados no contexto terapêutico, por exemplo, pode ser a autorrevelação de sentimentos do terapeuta ao cliente e a execução desse comportamento em um cenário de role-playing pode ser bastante prejudicada por se tratar de um contexto artificial. É salientado na literatura que a autorrevelação seja autêntica, sem o uso pelo terapeuta de qualquer tipo de simulação (Guenzen, 2014; Tsai, Plummer, Newring, \&, Kohlenberg, 2010). Além disso, para fazer uma autorrevelação, o terapeuta deve estar sob controle de seus próprios sentimentos em relação ao cliente (Guenzen, 2014; Knott et al., 2015). É possível que esse comportamento tenha sua emissão prejudicada num contexto artificial, onde não há uma história real entre terapeuta e cliente. Assim, fica o questionamento: os participantes não desenvolveram esse comportamento no treino ou não tiveram ocasião adequada para emiti-lo?

Pode haver também problemas em relação à avaliação do comportamento de reforçar a emissão de CIVPs pelo cliente nos cenários de role-playing. Esse comportamento pode ter sido de difícil per- 
cepção pelos juízes, uma vez que foram trabalhadas no treino algumas topografias sutis para a sua emissão, como expressões com o olhar. Além disso, topografias mais visíveis de reforçamento, como uma fala do terapeuta, podem soar artificiais - sobretudo no cenário de role-playing.

O comportamento de dirigir ações sob controle das variáveis relevantes do aqui/agora da sessão do contexto terapêutico, por sua vez, foi o mais desenvolvido pelos participantes do estudo. Isso pode ter acontecido por ele ser um comportamento de fácil identificação, estritamente verbal e passível de ser aplicado num contexto artificial.

A análise dessas possíveis variáveis que podem ter influenciado os resultados permitiu o desenvolvimento de uma conclusão pertinente aos estudos sobre treinos de terapeutas FAP. Tais estudos avaliaram de maneira negativa a medida dos resultados dos treinos por meio de medidas de autorrelato (Kanter et al., 2012), indicando como mais desejáveis as medidas do comportamento do terapeuta no contexto terapêutico (Kanter et al., 2012; Knott et al., 2015) e o uso de cenários de role-playing como possíveis alternativas, caso a medida do comportamento do terapeuta no contexto terapêutico seja inviável (Fairburn \& Cooper, 2011; Kanter et al., 2012).

Acreditamos, contudo, que o método de avaliação dos resultados de um treino de terapeutas depende do comportamento a ser treinado. O role-playing pode ser uma alternativa viável e efetiva, como indicaram Fairburn e Cooper (2011) e Kanter et al. (2012), mas para alguns comportamentos ele pode não ser o contexto ideal. É recomendável, portanto, que um treino de várias habilidades terapêuticas utilize mais de uma medida para verificar seu efeito no repertório comportamental de seus participantes.

\section{Considerações Finais}

Os resultados do presente estudo indicaram que o treino de comportamentos de intimidade para terapeutas FAP é capaz de produzir melhoras no repertório ligado ao "saber que" dos participantes, especialmente na capacidade de sistematizar comportamentos de intimidade que o terapeuta FAP pode emitir no contexto terapêutico. Após o treino, os participantes foram capazes de identificar adequadamente comportamentos de intimidade do terapeuta FAP no contexto terapêutico e descrever esses comportamentos funcionalmente. $\mathrm{O}$ treino promoveu também a capacidade dos participantes de diferenciar comportamentos que representam fatores terapêuticos comuns de comportamentos de intimidade do terapeuta FAP.

Não foi possível verificar mudanças nos comportamentos de intimidade dos participantes, havendo apenas indícios de que o comportamento de dirigir ações sob controle das variáveis relevantes do aqui/agora da sessão do contexto terapêutico pode melhorar. Contudo, acredita-se que uma réplica desse treino com as variações metodológicas sugeridas possa obter resultados diferentes.

O estudo teve limitações quanto ao número de participantes e quanto ao fato de ter verificado apenas os resultados imediatos do treino. Assim, não foi possível realizar o cálculo de concordância entre os juízes sobre o desempenho dos participantes e não há clareza sobre se as eventuais melhoras obtidas pelos participantes se mantêm ao longo do tempo. Além disso, o estudo apresentou limitações quanto à forma com a qual os comportamentos passíveis de aplicação pelo terapeuta em sessão foram avaliados. Apesar dessas limitações, os resultados ressaltam que o treino de comportamentos de intimidade para terapeutas é viável e promissor.

Sugere-se que estudos futuros apliquem o treino de intimidade proposto nesse trabalho para um número maior de participantes, a fim de possibilitar análises estatísticas que revelem se as eventuais mudanças são significativas. Sugere-se também que sejam utilizadas medidas de seguimento (follow-up) após o treino, a fim de verificar se as mudanças observadas se mantêm ao longo do tempo. Além disto, parece importante que o treino seja aplicado a participantes com diferentes graus de formação - estudantes de psicologia com e sem experiência clínica, terapeutas com pouco e muito tempo de experiência e etc. - a fim de melhor avaliar e comparar seus efeitos. 


\section{Referências}

Abbey, R. D., Clopton, J. R., \& Humphreys, J. D. (2007). Obsessive-compulsive disorder and romantic functioning. Journal of Clinical Psychology, 63, 1181-1192.

Callaghan, G. M. (2006). The Functional Idiographic Assessment Template (FIAT) System. The Behavior Analyst Today, 7(3), 357398.

Callaghan, G. M., \& Follette, W. C. (2008). Coding Manual for the Functional Analytic Psychotherapy Rating Scale (FAPRS). The Behavior Analyst Today, 9, 57-97.

Cordova, J. V., \& Scott, R. L. (2001). Intimacy: a behavioral interpretation. The Behavior Analyst, 24 (1) 75-86.

Fairburn, C. G., \& Cooper, Z. (2011). Therapist competence, therapy quality, and thrapis training. Behavior Research and Therapy, 49, 373378. Doi:10.1016/j.brat.2011.03.005

Freitas, S. T. (2011). Efeitos de procedimentos focados na relação terapêutica sobre comportamentos geralmente descritos nos quadros de depressão. Dissertação de Mestrado. Programa de Pós-Graduação em Psicologia. Universidade Federal do Paraná. Curitiba, PR.

Foa, E. B., Huppert, J. D., Leiberg, S., Langner, R., Kickic, R., Gajcak, G., et al. (2002). The obsessive-compulsive inventory: Development and validation of a short version. Psychological Assessment, 14, 485-496.

Follette, W. C., \& Callaghan, G. M. (1995). Do as I do, not a I say: a behavioral-analytic approach to supervision. Professional Psychology: research and practice, 26(4), 413-421.

Fugita, L. M. P. (2014). Elaboração de um mapa de ensino do comportamento de intimidade de terapeutas da Psicoterapia Analítica Funcional. Monografia não publicada. Universidade Federal do Paraná. Curitiba, PR.

Guenzen, L. C., \& Silveira, J.M. (2013). Intimidade na relação terapêutica: Uma caracterização da palavra por terapeutas analítico-comportamentais. Psicologia Argumento, 31 (74), 547-559.

Guenzen, L. C. (2014). Os possíveis efeitos do comportamento de autorrevelação do terapeuta analítico-comportamental em um processo te- rapêutico. Dissertação de Mestrado. Programa de Pós-Graduação em Psicologia. Universidade Federal do Paraná. Curitiba, PR.

Haworth, K., Kanter, J.W., Tsai, M., Kuczynski, A. M., Rae, J. R., \& Kohlenberg, R. J. Reinforcement matters: A preliminary, laboratory-based component-process analysis of Functional Analytic Psychotherapy's model of social connection. Journal of Contextual Behavioral Science (2015), http://dx.doi.org/10.1016/j.jcbs.2015.08.003.

Kanter, J. W., Tsai, M., Holman, G., \& Koerner, K. (2012). Preliminary data from a randomized pilot study of web-based Functional Analytic Psychotherapy Therapist Training. Psychotherapy, 50 (2), 248 -255. doi: 10.1037/ a0029814.

Knott, L. E., Wetterneck, C. T., Derr, D., \& Tolentino, R. (2015). A functional analytic perspective of therapist intimacy in and out of session. International Journal of Behavioral Consultation and Therapy, 9 (4), 6-10.

Kohlenberg, R. J., \& Tsai, M. (2004). Psicoterapia analítica functional: criando relações terapêuticas intensas e curativas (R. R. Kerbauy, Org.). Santo André, ESETec Editores Associados. (Trabalho original publicado em 1991)

Kohlenberg, R. J., Kohlenberg, B., \& Tsai, M. (2011). Intimidade. In Tsai, M. et al. (Orgs.), Um guia para a psicoterapia analítica funcional (FAP): consciência, coragem, amor e behaviorismo (pp. 171-186). Santo André: ESETec (Obra original publicada em 2009).

Lepienski, A. F. (2015). Relações entre uma supervisão em Psicoterapia Analítico Funcional e as respostas de uma terapeuta e sua cliente em sessão. Dissertação de Mestrado. Programa de Pós-Graduação em Psicologia. Universidade Federal do Paraná. Curitiba, PR.

Mangabeira, V. C. S. (2014). Intervenções sinalizadas e não-sinalizadas da psicoterapia analítica funcional sobre comportamentos clinicamente relevantes. Tese de Doutorado. Instituto de Psicologia. Universidade de São Paulo. São Paulo, SP.

Meurer, P. H. (2011). Efeito da apresentação de feedback no comportamento do terapeuta de evocar e responder aos comportamentos clinicamente relevantes. Dissertação de Mestrado. 
Programa de Pós-Graduação em Psicologia. Universidade Federal do Paraná. Curitiba, PR.

Newth, S. \& Rachman, S. (2001). The concealment of obsessions. Behaviour Research and Therapy, 39, 457-464.

Oshiro, B., Kanter, J., \& Meyer, S. (2012). A single-case experimental demonstration of Functional Analytic Psychotherapy with two clients with severe interpersonal problems. International Journal of Behavioral Consultation and Therapy, 7(2-3), 111-117.

Patten, S. B. (1991). Are the Brown and Harris "vulnerability factors" risk factors for depression?. Journal of Psychiatric Neuroscience, 16, 267-271.

Schaefer, M. T., \& Olson, D. H. (1981).Assessing intimacy: The Pair Inventory. Journal of Marital and Family Therapy, 7, 47-60.

Silva-Dias, A. Y. M. (2015). Efeitos da modelagem e da modelação do comportamento vulnerável à punição com um casal em terapia analítico- comportamental. Dissertação de mestrado. Programa de Pós-Graduação em Psicologia da Universidade Federal do Paraná. Curitiba, PR.

Silva-Dias, A. Y. M., \& Silveira, J. M. (2016). Comparação de duas intervenções no tratamento de um casal: o treino do comportamento vulnerável à punição. Revista Acta Comportamentalia, 24 (1), 61-77.

Silveira, J. M., Callaghan, G. M., Stradioto, A., Maeoka, B. E., Maurício, M. N., \& Goulin, P. (2009). Efeitos de um treino em Psicoterapia Analítica Funcional sobre a identificação feita pelo terapeuta de comportamentos clinicamente relevantes de seu cliente. Revista Brasileira de Terapia Comportamental e Cognitiva, 9(2), 346365. Retrieved from http://www.usp.br/rbtcc/ index.php/ RBTCC/ article/view/409/303

Sousa, A. C. A. de, \& Vandenberghe, L. (2007) Possibilidades da FAP como método de supervisão de terapeutas com clientes Boderlines. Revista Brasileira de Terapia Comportamental e Cognitiva, 9(1), 1-11.

Sprecher, S., Treger, S., Wondra, J. D., Hilaire, N., \& Wallpe, K. (2013). Taking turns: Reciprocal self-disclosure promotes liking in initial interactions. Journal of Experimental Social Psychology, 49(5), 860-866. doi:10.1016/j. jesp.2013.03.017
Stemberger, R. M. T., Thomas, A. M., Mansueto, C. S., \& Carter, J. G. (2000). Personal toll of trichotillomania: Behavioral and interpersonal sequelae. Journal of Anxiety Disorders, 14, 97-104.

Tsai, M., Kohlenberg, R. J., Kanter, J. W., Kohlenberg, B., Follette, W. C., \& Callaghan, G. M. (2011). Um guia para a psicoterapia analítica functional (FAP): consciência, coragem, amor e behaviorismo (F. Conte, \& M. Z. Brandão). Santo André: ESETEc Editores Associados. (Obra publicada originalmente em 2009).

Tsai, M., Callaghan, G. M., Kohlenberg, R. J., Follette, W. C., \& Darrow, S. M. (2011). Supervisão e desenvolvimento pessoal do terapeuta. In M. Tsai, R. J. Kohlenberg, J. W. Kanter, B. Kohlenberg, W. C. Follette, \& G. M. Callaghan (Orgs). Um guia para a Psicoterapia Analítica Funcional: consciência, coragem, amor e behaviorismo (pp. 211 - 247). Santo André: ESETEC Editores Associados. (Obra publicada originalmente em 2009).

Tsai, M., Plummer, M. D., Kanter, J. W., Newring, R. W., \& Kohlenberg, R. J. (2010). Therapist Grief and Functional Analytic Psychotherapy: Strategic Self-Disclosure of Personal Loss. J Contemp Psychother, 40, 1-10.

Tsai, M., Callaghan, G. M., \& Kohlenberg, R. J. (2013). The use of awareness, courage, therapeutic love, and behavioral interpretation in Functional Analytic Psychotherapy. Psychotherapy, 50 (3), 366-370.

Vandenberghe, L., \& Pereira, M. B. (2005). O papel da intimidade na relação terapêutica: uma revisão teórica à luz da análise clínica do comportamento. Psicologia: Teoria e Prática, 7 (1), 127-136.

Villas-Bôas, A. A. (2015). Efeitos diferenciais de análise das contingências intra e extrassessão em terapia analítica funcional. Tese de Doutorado. Instituto de Psicologia. Universidade de São Paulo. São Paulo, SP.

Wetterneck, C. T., \& Hart, J. M. (2012). Intimacy is a Transdiagnostic Problem for Cognitive Behavior Therapy: Functional Analytic Psychotherapy is a solution. International Journal of Behavioral Consultation and Therapy, 7(2-3), 167-176. doi.org/10.1037/h0100956. 
Wielenska, R. C., \& Oshiro, C. K. B. (2012). FAP group supervision: reporting educational experiences at the University of São Paulo, Brazil. International Journal of Behavioral Consultation and Therapy, 7 (2-3), 177 - 181. Retrieved from http://www.baojournal.com/IJBCT/ IJBCT-7_2-3/7_2-3_Articles/A25.pdf

Informações do Artigo

Histórico do artigo:

Submetido em: 07/03/2016

Primeira decisão editorial: 13/07/2016

Aceito em: 10/08/2016

Editor Associado: Jan Leonardi 